\title{
Defining Early Multidisciplinary Goals: NEXTO Trial in High-Risk Colorectal Cancer with Liver Metastases
}

\author{
Jeremy D. Kratz, $\mathrm{MD}^{\mathbf{1}}$, and Noelle K. LoConte, $\mathrm{MD}^{1,2}$ \\ ${ }^{1}$ Division of Hematology and Oncology, Department of Medicine, University of Wisconsin-Madison, Madison, WI; \\ ${ }^{2}$ University of Wisconsin Carbone Cancer Center, University of Wisconsin-Madison, Madison, WI
}

The current issue of Annals of Surgical Oncology includes a report by Mise et al. reporting results of the NEXTO trial. ${ }^{1}$ Controlling distant micrometastatic disease remains a critical challenge in the management of advanced colorectal cancer (CRC). For locally advanced $\mathrm{CRC}$, the rates of locoregional recurrence remain quite low at $4.4 \%$ for colon and $11.2-12.1 \%$ for rectal cancer. ${ }^{2,3}$ Metastatic disease by definition requires hematogenous dissemination with loss of systemic disease control. Prior management by primary resection and adjuvant chemotherapy resulted in a $40 \%$ rate of disease recurrence, yet a relatively modest risk of approximately $4 \%$ for marginal recurrence. ${ }^{4}$ FOLFOX chemotherapy (5-fluorouracil and oxaliplatin with leucovorin rescue) has remained a standard-of-care treatment regimen in metastatic CRC. This study examined FOLFOX and the addition of anti-epidermal growth factor receptor (EGFR) inhibition with cetuximab prior to surgical consideration in advanced CRC with hepatic metastases to evaluate conversion to $\mathrm{R} 0$ resection.

A major strength of this study is the emphasis placed on multidisciplinary evaluation and care, specific in the early management of patients under consideration for metastasectomy. ${ }^{1}$ It has been consistently shown that multidisciplinary care in colorectal cancer including surgery, medical oncology, radiation oncology, radiology, and pathology can reduce time to initiation of therapy and expedite preoperative surgical evaluation. ${ }^{5}$ Patients who

(C) Society of Surgical Oncology 2020

First Received: 8 May 2020;

Published Online: 22 May 2020

J. D. Kratz, MD

e-mail: jkratz@uwhealth.org undergo metastasectomy have shown significant differences in outcomes from registry reports with 5-year overall survival of $37 \%$ (vs. $2 \%$ for those deemed ineligible). ${ }^{6}$ The NEXTO study incorporated molecular profiling with KRAS wildtype status, necessitating expedited molecular profiling in alignment with standardized guideline-based practice. ${ }^{7}$ The expertise of surgeons who specialize in hepatectomy is of great value to a treating medical oncologist in defining the goals of systemic therapy and optimizing the timing of operative management. The ability to convert high-risk metastatic CRC to achieve successful R0 resection reflects optimized delivery of team-based cancer care.

The NEXTO trial leveraged broad inclusion criteria with extrahepatic disease burden. This population was of particular high risk as the number of metastatic sites has been predictive of overall survival in CRC. ${ }^{8}$ Surgical margin status (R0 versus R1) in the liver is predictive of survival after hepatic resection. ${ }^{4}$ For patients with concurrent hepatic and pulmonary metastases, an increasing frequency of pulmonary metastases $(>1)$ has shown inferior 5-year overall survival of $32.2 \%$ versus $55.4 \%$ for patients with single sites of pulmonary metastases. ${ }^{9}$ The feasibility of synchronous primary resections has been well established for patients with limited foci of extrahepatic metastases. ${ }^{10}$ Understanding the features of extrahepatic metastatic burden in the NEXTO study including bulk at these sites would help in understanding the feasibility of conversion therapy to achieve R0 resection for this high-risk population. The NEXTO study supports a generalized strategy of systemic therapy using standard-of-care neoadjuvant chemotherapy followed by multidisciplinary procedural evaluation with expanded subspecialty expertise to achieve complete resection. 
The study population included patients with high-risk hepatic metastases defined as $\geq 5$ lesions ( $n=10,21 \%)$ and/or those with previously established criteria for unresectable disease ${ }^{11}(n=38,79 \%)$. The definitions of resectability are consistent with modern approaches directed at assessing conversion hepatectomy. ${ }^{12}$ However, including $21 \%$ of patients with technically resectable disease makes it difficult to interpret the outcome of this study due to mixed inclusion in comparison with contemporary trials. Interestingly, investigations of resectable CRC with hepatic metastases from the phase III New EPOC trial revealed inferior overall survival for neoadjuvant FOLFOX plus cetuximab at 55.4 months versus 81.0 months with FOLFOX alone. ${ }^{13}$ The authors suggest a nonstatistical difference between technically resectable and unresectable disease, however the certainty of this finding must be contextualized to the limited power of post hoc subgroup analysis.

Future trial design must consider the spectrum of clinical presentations from upfront resectable, to potentially conversion of unresectable disease. The study design with primary endpoint of $\mathrm{R} 0$ resection predefines a population referred for surgical consultation. This study population differs from investigations of trials which escalate systemic therapy, where R0 resection is included in secondary or experimental outcomes and practically led by medical oncology. In the RAS/RAF wildtype population of unresectable disease, escalation of chemotherapy to FOLFOXIRI and cetuximab in the phase II MACBETH trial led to $35 \%$ of patients undergoing metastatectomy. ${ }^{14}$ A significant limitation in understanding the NEXTO trial is the context of definitions of high-risk metastatic disease with mixed differences between unresectable and technically resectable disease.

The development of targeted therapeutics (i.e., EGFR inhibition) requires an understanding of underlying disease biology to define meaningful clinical outcomes. The prognostic utility of $\mathrm{R} 0$ resection on long-term overall survival was established using adjuvant cytotoxic chemotherapy (FOLFOX) prior to the development of targeted therapies. ${ }^{4,10}$ The results of incorporating targeted therapy have been of concern, specific to the incorporation of EGFR inhibition. The New EPOC trial used a similar intervention for resectable disease including EGFR inhibition (cetuximab) in combination with FOLFOX chemotherapy. The addition of EGFR inhibition resulted in inferior overall survival, specifically a worsened outcome in post progression survival for patients in the arm receiving neoadjuvant EGFR inhibition at 23.5 months versus 33.5 months in FOLFOX control. ${ }^{13}$ The inferior post progression-free survival suggested more aggressive disease biology after operative management with prior exposure to EGFR inhibition. The expression of c-Myc was enhanced in patients receiving EGFR inhibition ${ }^{15}$ as an exploratory endpoint of the New EPOC trial. Enhanced expression of c-Myc predicted inferior clinical outcomes, suggesting a mechanism of resistance specific to the use of EGFR inhibition in the neoadjuvant setting. While the additional of EGFR inhibition may provide radiographic response to achieve surgical conversion, this needs to be weighed against a biologic basis of potential harm through selection of subclonal resistance resulting in inferior control of micrometastatic disease. Potential harm observed in the New EPOC trial makes escalation with EGFR inhibition challenging to support in populations with technically resectable disease.

Biomarkers predictive of response to EGFR inhibition in colorectal cancer continue to develop in the metastatic setting and should be included to characterize the study population. Extended spectrum RAS alterations (KRAS and NRAS at exons 2, 3, and 4) as well as BRAF V600E have predicted worsened outcomes for the addition of EGFR inhibition to front-line FOLFOX chemotherapy. ${ }^{16}$ While representing $<5 \%$ of the overall population, emerging biomarkers such as HER2 amplification have also been predictive of clinical resistance to EGFR inhibition. $^{17}$

Beyond a given molecular alteration, clinical characteristics have also predicted differences in clinical outcomes specific to the use of EGFR inhibition. In the metastatic setting, SWOG/CALGB80405 revealed that the site of the primary tumor predicted worsened outcomes for EGFR inhibition for cancers arising proximal to the splenic flexure (right-sided) with reduced response rates and survival. $^{18}$ Additionally, the role of disease bulk should be considered when assessing the benefit of EGFR inhibition across lines of therapy. It is established that patients with right-sided tumors have delayed time to clinical presentation and often present with increased metastatic bulk. ${ }^{19}$ In patients with single sites of metastases greater than $35 \mathrm{~mm}$ (defined by median diameter of hepatic metastases), we recently observed lower response rates and overall survival for the use of EGFR inhibition. ${ }^{20}$ Thus, primary tumor sidedness and disease bulk are emerging as clinical biomarkers of therapeutic resistance to EGFR inhibition (Fig. 1).

The NEXTO trial provides hope in progress for the management of high-risk metastatic colorectal cancer through comprehensive multidisciplinary care. Understanding differences in disease biology afforded by advances in molecular profiling and clinical presentation should guide future prospective trial design for neoadjuvant strategies to achieve $\mathrm{R} 0$ resection. The improvement in multidisciplinary teams is invaluable in setting goals, specific to trial strategies that escalate the use of systemic therapy. Therapeutic strategies which incorporate targeted 
FIG. 1 Clinical presentation of colorectal cancer by primary sidedness: a right-sided tumor at hepatic flexure with large primary tumor and multifocal sites of metastases, b left-sided primary at splenic flexure with limited nonbulky sites of hepatic metastases
(A) Right-Sided Primary

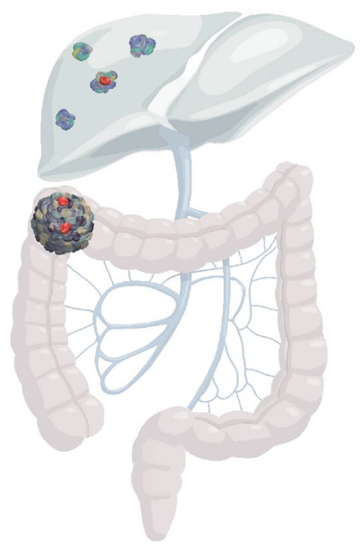

1) Delayed clinical presentation

2) Higher likelihood of bulky metastases

3) Increase clonal RAS/RAF alterations

4) RAS $^{W T} / R^{\prime} A F^{W T}$ CRC resistant to EGFRi
(B) Left-Sided Primary

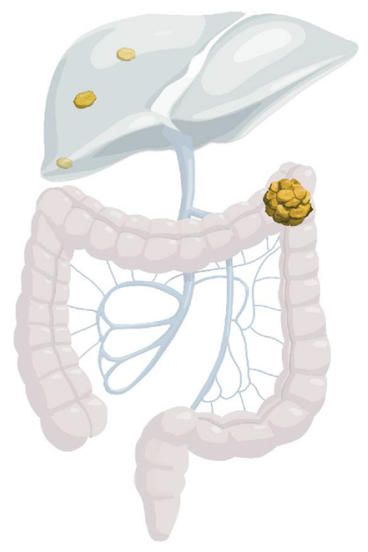

1) Early clinical presentation with hematochezia

2) RAS/RAF alterations less frequent

3) Improved clinical outcomes with EGFRi therapies should continue to expand the set of tools for biomarkers to aid in appropriate patient selection. For EGFR inhibition, these should incorporate tumor characteristics including primary sidedness, metastatic disease bulk, and distinct molecular selection extrapolated from our understanding in the unresectable disease setting. These strategies should also look ahead to the incorporation of ctDNA as correlative biomarkers over the course of neoadjuvant therapy to track dynamic change while assessing systemic disease control. ${ }^{21}$ The importance of team-based oncology underlies the mission and execution of work to advance clinical outcomes in the practice of modern oncologic care.

ACKNOWLEDGMENT The authors would like to thank David L. Porter for support in graphic design provided in this manuscript.

AUTHOR CONTRIBUTIONS Wrote the manuscript: JDK., NKL.

FINANCIAL SUPPORT This project was supported by P30 CA014520 (CCSG Cancer Center Support Grant, University of Wisconsin Carbone Cancer Center). The authors thank the University of Wisconsin Biology of Aging and Age-Related Diseases T32 Training Grant \#5T32AG000213-28 for providing salary support for J.D.K.

DISCLOSURE J.D.K. none, N.K.L. has served as consultant for Abbvie and advisory boards for Celgene and Bayer.

\section{REFERENCES}

1. Mise Y, Hasegawa K, Saiura A, et al. A multicenter phase II trial to evaluate the efficacy of mFOLFOX6 + cetuximab as induction chemotherapy to achieve R0 surgical resection for advanced colorectal liver metastases (NEXTO trial). Ann Surg Oncol. 2020. (in press)

2. Liska D, Stocchi L, Karagkounis G, et al. Incidence, patterns, and predictors of locoregional recurrence in colon cancer. Ann Surg Oncol. 2017;24(4):1093-9.

3. Allegra CJ, Yothers G, O'Connell MJ, et al. Final results from NSABP protocol R-04: Neoadjuvant chemoradiation (RT) comparing continuous infusion (CIV) 5-FU with capecitabine (Cape) with or without oxaliplatin (Ox) in patients with stage II and III rectal cancer. Am Soc Clin Oncol. 2014.

4. Pawlik TM, Scoggins CR, Zorzi D, et al. Effect of surgical margin status on survival and site of recurrence after hepatic resection for colorectal metastases. Ann Surg. 2005;241(5):715.

5. Kozak VN, Khorana AA, Amarnath S, Glass KE, Kalady MF. Multidisciplinary clinics for colorectal cancer care reduces treatment time. Clin Colorectal Cancer. 2017;16(4):366-71.

6. Segelman J, Singnomklao T, Hellborg H, Martling A. Differences in multidisciplinary team assessment and treatment between patients with stage IV colon and rectal cancer. Colorectal Dis. 2009;11(7):768-74.

7. Levine RA, Chawla B, Bergeron S, Wasvary H. Multidisciplinary management of colorectal cancer enhances access to multimodal therapy and compliance with National Comprehensive Cancer Network (NCCN) guidelines. Int $J$ Colorectal Dis. 2012;27(11):1531-8.

8. Loh KWJ, Shapiro JD, Tran B, et al. Tumor burden (TB) as a prognostic indicator in patients with metastatic colorectal cancer (mCRC). Am Soc Clin Oncol; 2014.

9. Sclafani F, Incarbone M, Rimassa L, et al. The role of hepatic metastases and pulmonary tumor burden in predicting survival after complete pulmonary resection for colorectal cancer. $J$ Thoracic Cardiovasc Surg. 2013;145(1):97-103.

10. Elias D, Sideris L, Pocard M, et al. Results of R0 resection for colorectal liver metastases associated with extrahepatic disease. Ann Surg Oncol. 2004;11(3):274-80.

11. Beppu T, Miyamoto Y, Sakamoto Y, et al. Chemotherapy and targeted therapy for patients with initially unresectable colorectal liver metastases, focusing on conversion hepatectomy and longterm survival. Ann Surg Oncol. 2014;21(3):405-13.

12. Folprecht G, Gruenberger T, Bechstein W, et al. Survival of patients with initially unresectable colorectal liver metastases 
treated with FOLFOX/cetuximab or FOLFIRI/cetuximab in a multidisciplinary concept (CELIM study). Ann Oncol. 2014;25(5):1018-25.

13. Bridgewater JA, Pugh SA, Maishman T, et al. Systemic chemotherapy with or without cetuximab in patients with resectable colorectal liver metastasis (New EPOC): long-term results of a multicentre, randomised, controlled, phase 3 trial. Lancet Oncol. 2020;21(3):398-411.

14. Cremolini C, Antoniotti C, Lonardi S, et al. Activity and safety of cetuximab plus modified FOLFOXIRI followed by maintenance with cetuximab or bevacizumab for Ras and BRAF wild-type metastatic colorectal cancer: a randomized phase 2 clinical trial. JAMA Oncol. 2018;4(4):529-36.

15. Pugh S, Thiébaut R, Bridgewater J, et al. Association between miR-31-3p expression and cetuximab efficacy in patients with KRAS wild-type metastatic colorectal cancer: a post hoc analysis of the New EPOC trial. Oncotarget. 2017;8(55):93856.

16. Douillard J-Y, Oliner KS, Siena S, et al. Panitumumab-FOLFOX4 treatment and RAS mutations in colorectal cancer. $N$ Engl J Med. 2013;369(11):1023-34.

17. Wang G, He Y, Sun Y, et al. Prevalence, prognosis and predictive status of HER2 amplification in anti-EGFR-resistant metastatic colorectal cancer. Clin Transl Oncol. 2019:1-10.
18. Venook AP, Niedzwiecki D, Innocenti F, et al. Impact of primary $\left(1^{\circ}\right)$ tumor location on overall survival (OS) and progression-free survival (PFS) in patients (pts) with metastatic colorectal cancer (mCRC): Analysis of CALGB/SWOG 80405 (Alliance). Am Soc Oncol; 2016.

19. Benedix F, Kube R, Meyer F, et al. Comparison of 17,641 patients with right-and left-sided colon cancer: differences in epidemiology, perioperative course, histology, and survival. Dis Colin Rectum. 2010;53(1):57-64.

20. Kratz JD, Uboha NV, Lubner SJ, et al. Metastatic bulk independently predicts outcomes for EGFR precision targeting in colorectal cancer. $J$ Natl Comp Cancer Netw. 2018;16(12):1442-50

21. Strickler JH, Loree JM, Ahronian LG, et al. Genomic landscape of cell-free DNA in patients with colorectal cancer. Cancer Discov. 2018;8(2):164-73.

Publisher's Note Springer Nature remains neutral with regard to jurisdictional claims in published maps and institutional affiliations. 